\title{
Energy Efficient and VM Consolidation Framework using Improved Spider Monkey Optimization Algorithm
}

\author{
Kethavath Prem Kumar, Thirumalaisamy Ragunathan, Devara Vasumathi
}

\begin{abstract}
Cloud Computing is rapidly being utilized to operate informational technological services by outstanding technologies for a variety of benefits, including dynamically improved resources planning and a new service delivery method. The Cloud computing process is occurred by allowing the client devices for data access through the internet from a remote server, computers, and the databases. An internet connection is linked among the front end users such as client device, network, browser, and software application with the back end that constitutes of servers, computers, and database. For satisfying the demands of the Service Level Agreement (SLA), providers of cloud service should reduce the usage of energy. Capacity reservations oriented system is available by clouds' providers to permit users for customizing Virtual Machines (VMs) having specified age and geographic resources, reduces the amount to be paid for cloud services. To overcome the aforementioned issue, an Improved Spider Monkey Optimization (ISMO) approach is proposed for cloud center optimization. The VM consolidation architecture based on the proposed ISMO algorithm decreases energy usage while attempting to prevent Service Level Agreement breaches. The accessibility of hosts or virtual machines (VMs) for task performance is measured by fitness. If the number of tasks to be handled increases the hosts of VMs available at right state. The proposed VM consolidation architecture decreases energy usage while also attempting to prevent Service Level Agreement breaches and also provide energy-efficient computing in data centers. The proposed approach may be utilized to provide energy-efficient computing in data centers. The energy efficiency of the proposed ISMO method is achieved 28266 whereas, the existing algorithm showed an energy efficiency of 6009 and 10001.
\end{abstract}

Keywords: Cloud Computing, Information Technology, Service Level Agreement, Improved Spider Monkey Optimization, VM consolidation.

Manuscript received on August 20, 2021.

Revised Manuscript received on August 26, 2021.

Manuscript published on September 30, 2021.

* Correspondence Author

Kethavath Prem Kumar*, Research Scholar, ACE Engineering College, Department of Computer Science \& Engineering, Hyderabad, India.Email: kpremkumarcs@gmail.com

Thirumalaisamy Ragunathan, Ph.D, SRM University, Department of Computer Science \& Engineering, Amaravathi, India. Email: ragunathan.t@srmap.edu.in

Devara Vasumathi, Ph.D, Jawaharlal Nehru Technological University, Department of Computer Science \& Engineering, Hyderabad, India. Email: asukumar_devara@jntuh.ac.in

(c) The Authors. Published by Blue Eyes Intelligence Engineering and Sciences Publication (BEIESP). This is an open access article under the CC BY-NC-ND license (http://creativecommons.org/licenses/by-nc-nd/4.0/)

\section{INTRODUCTION}

Cloud computing allows the system to have customization Resources on a required network like networks, storage and applications [1]. Cloud computing permits people and offices to make use of services like data, compute, storage and application. Companies like Microsoft, Salesforce, IBM, Amazon are trying for building advanced data center round the world for their clients [2]. Cloud supplies Infrastructure as a Service (IaaS), Software as a service (SaaS), and Platform as a Service (PaaS) for consumers using via internet accesses for the providers [3]. The IaaS model, cloud data centers consist of many VMs by a single PM with the help of virtualization technology and IaaS suppliers provide compute storage and memory resources to the cloud clients. Clients pay to IaaS suppliers based on the size and hour of using the Virtual Machine [VM] facility [4]. The cloud data centers are rapidly increasing, and the cost of functioning is increasing because of the high energy usage and habitat pollutants of the data center [5]. A huge amount of electricity is consumed and greenhouse gas like $\mathrm{CO} 2$ are emitted into the environment due to the data centers which contain plenty of physical machines such as hosts or servers [6].

The Green cloud computing technology is used for handling an efficient data and accomplishing them to secure the resources. It also lowers energy utilization which boosts the growth of cloud computing facilities. An increase in Network (or client) devices communicating with the application, prevailing User Interface, Software, Service Cloud Runtime, Storage, Infrastructure, Management, Security data center in cloud computing result in high energy consumption [7]. VM problem placement is the process that decides the finest PM to load a VM, hence this is also called NP-hard (Non-deterministic Polynomial-time) problem because finding a solution to this problem is hard. It is defined as a bin-packing problem which will be similar to the VMs that are loaded into PMs [8]. There are several limitations in VM consolidation of cloud computing - Few VMs will not be able to get their necessary resources due to variable application's caseload operating on the VMs high electrical power demand. The issue holds the application's Quality of Service(QoS) and increases the time of reaction or non-success (or downtime) [9]. It's reported that significant space of data centers is unused because only $10-30 \%$ of large data centers are utilized [10]. For solving this problem, ISPMO is proposed for cloud center optimization.

Published By:

Blue Eyes Intelligence Engineering and Sciences Publication

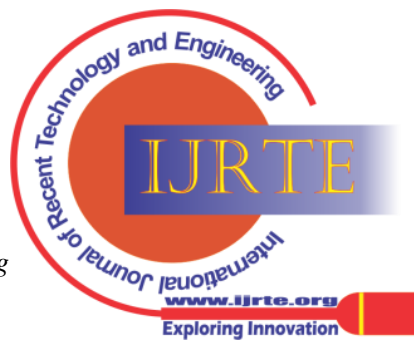


The proposed VM consolidation architecture decreases energy usage while also attempting to prevent Service Level Agreement breaches and also provide energy-efficient computing in data centers.

The presented research work is organized as follows: The literature review is discussed under Section 2 and section 3 describes about the proposed methodology. The section 4 describes the experimental results, discussion and section 5 explains about the conclusion for the proposed method.

\section{LITERATURE REVIEW}

The existing methods based on data centers optimization in cloud computing is reviewed in this section. Also, the advantages and limitations of existing reviewed methods are described in this section,

Jialei LIU et al. [11] developed a green cloud computing consolidation for prediction. The developed model reduced power consumption while maintaining SLAs in Containers-as-a-Service data centers. SLA violations, containers movement, consumption of resources as well as the mean of live VMs are all decreased in this architecture, But, the SLA-driven consolidating technique did not look for the overbooking factors from other resources such as network and memory. However, it did not dynamically define the overbooking exploited factors based on the online polices estimated the container based applications.

Niloofar Gholipour et al. [12] created a cloud resource control strategy based on a multi-condition judgment mechanism that concurrently benefits from VM and Return On Investment (ROI) of cloud providers while Nonetheless, system characteristics such as RAM, Network Bandwidth, Memory, and Cooling were not taken into account.

Abdelhameed Ibrahim et al. [13] designed a major responsibility algorithm using particle swarm optimization. The devised model was successful in identifying the near-optimal position for the relocated VMs and minimizing the number of devices in the network of the hosts. However, when the number of overburdened servers grows, it becomes necessary to migrate VMs from overburdened hosts regularly, which breaches the SLA.

Lianpeng $\mathrm{Li}$ et al. [14] constructed reliable regression analysis forecasting models for SLA for enhancing energy efficiency in VMs consolidating for cloud data. Compared to current approaches, the proposed mechanism demonstrated an effective threshold level. However, the suggested technique did not minimize violations in SLA and increased energy usage.

Marwa Gamal et al. [15] developed an algorithm known as Osmotic Bio-Inspired Load Balancing which reduced the amount of VM, energy usage, and shutdown hosts during cloud computing. The main aim of the developed model was to suggest for the hybrid meta heuristic approach as it combined both the bio-inspired load balancing and osmotic behaviour algorithms gave rise for Osmosis Hybrid Artificial Bee and Ant Colony (OHBAC) optimization method. As compared to other techniques, OHBAC had a large number of SLA Violations Time per Active Host (SLATAH).

Nagma Khattar et al. [16] created cloud-based electricity and adjustable cutoff VM consolidation system. The based model that utilized SLA driven container for containers migrations. The proposed method enhanced the simultaneously lowering greenhouse gases emissions.

developed architecture was as general as it operated both for CPU and input/output heavy jobs. The suggested framework's efficacy is demonstrated utilizing real-world data from Planet Lab as well as their Cloudsim system. The suggested approach may be utilized to provide energy-efficient processing in cloud information centers.

\section{PROPOSED METHODOLOGY}

The block diagram of the proposed ISPMO algorithm for cloud center and VM optimization is given in figure 1.

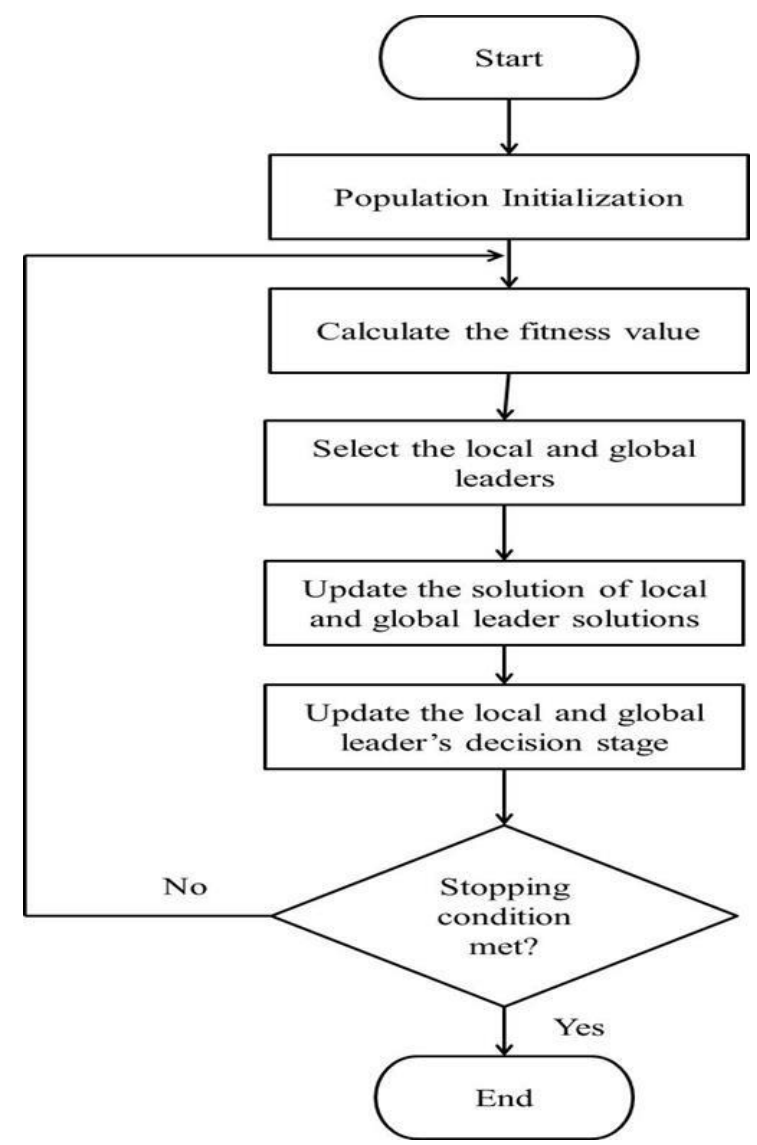

Figure I. The block diagram of proposed ISMO algorithm for VM consolidation.

Indeed, the Spider Monkey Optimization (SMO) technique is an environment-based optimization approach that was recently presented. In the area of swarm intelligent methods, SMO has been judged to be the best. The term "spider money" refers to a subspecies that belongs to the group of animals that have a controlled nuclear social hierarchy. These spider monkeys (SM) are always seen in bunches and exhibit clever searching behavior when looking for food. They make food hunting easier in a variety of approaches by exchanging relevant information with the different group members. The SMO algorithm was developed with influence by spider monkey's clever meal hunting technique. The Spider Monkeys' (SM) food hunting region represents the search area (hosts, VMs and the complete set of data centers) of the optimization issue (resource allocation problem).

Published By:

Blue Eyes Intelligence Engineering and Sciences Publication

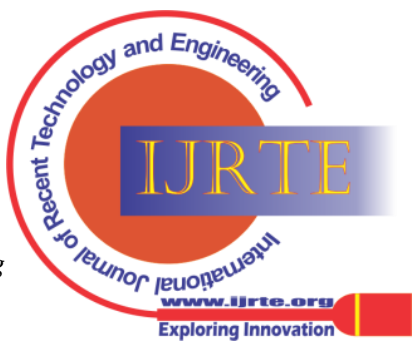


SMO algorithm contains a collection of communication rules for exchanging information and constant improvement (sharing data and continually learning regarding under- and over-utilization of VMs) to update locations in the whole search area. The VMs thresholding is performed on the basis of likelihood based on the processing tasks, the SMO method replaces its present location (recent VM - assigned with presence of tasks and a better one with the new VM has to be assigned). Four often used variables are perturbation speed, local leader restriction, leading global limit and highest number of individuals. SMO, like the other metaheuristic methods, starts by randomly placing uniformly produced spider monkeys (SM) which represent any locations of VMs or hosts.

Whenever there is zero progress throughout the global leader, the entire collection of host or VMs is divided into smaller groupings (global threshold limit). A council member is made up of possible VMs or hosts that belong to every one of the divided subgroups (local threshold limit) but, there is only one subgroup with the same local and global leaders. In advisement to an initialization stage, six adaptive steps are used to improve the allotment of presenters or VMs to inbound tasks: Global Leader Phase (GLP), Local Leader Phase (LLP), Learning Phase of Local Leader (LPLL), Learning Phase of Global Leader (LPGL), Deciding Phase of Global Leader (DPGL), and Deciding Phase of Local Leader following:

LLP: It is accountable for upgrading the present search area, which will be investigated in the following rounds. This location of hosts to update search region effectively. This trail location is calculated using the present role of the community politician, as well as a randomly picked object from that grouping. Furthermore, the potential dimensionality of the answers in the search area can be selected depending on the fluctuation value. Equation (1) is used to generate the newly built trail location.

$$
\begin{aligned}
S M_{P O S(N e w, j)}= & \left\{S M_{P O S i, j}+r(0,1)+\left(L L R_{P O S i, j}-S M_{P O S i, j}\right)+\right. \\
& r(-1,1) *\left(G L R_{P O S i, j}-S M_{P O S i, j}\right), \\
& \text { if } r(0,1) \geq P E R_{\text {rate }} S M_{P O S i, j} f r(0,1)<P E R_{\text {rate }}
\end{aligned}
$$

Where $r(0,1)$ is the fully special variable within the searching region for various research characteristics with the rate of disruption lower than the rate of disruption in the search region.

GLP: It is accountable for updating the current search space, much as the local leader stage. The procedure of improving the global leader phase is done uniquely. This phase is primarily concerned with the process of improving the search result using randomly chosen features. As a consequence, the probabilistic value obtained determines the likelihood of a spider monkey (hosts or VMs) being upgraded by a randomly selected single value from which is shown in equation (2).

$$
P R_{\text {select }}=0.9 *\left(\frac{F i t i}{M a x-F i t}\right)+0.1
$$

The trial point has the position will be regenerated on the basis of equation (3). (DPLL). The phases involved in SPMO are explained in the step creates a fresh trail location with each SM based on the

$$
\begin{aligned}
S M_{\mathrm{POSi}, j}=S M_{\mathrm{POSi}, j}+r(0,1) *( & \left(\operatorname{LLR}_{\mathrm{POSi}, j}-S M_{\mathrm{POS}, j}\right)+ \\
& r(-1,1) *\left(S M_{\text {Randomi }, j}-S M_{\mathrm{POSi}, j}\right)
\end{aligned}
$$

The fitness values of current hosts or VMs are then contrasted towards the newly produced positions of sites or VMs in an attempt to select the best candidate from the search area for the prospective application procedure.

LPGL: If a spider monkey's (host or VM) location seems to have a higher fitness value than all others primates (hosts or VMs) within the community area, that monkey would be the Global Leader throughout this stage. In addition, if no change is seen in the selected global leader place, the Global Limit Trial (GLT) is increased by one. GLT is an important component in keeping track of how many rounds the global leader hasn't been changed.

LPLL: During this step, the greedy choice is used to pick community leaders for every and every member state, similar to how the global powerhouse is chosen. The local limitation count is also increased if the specified local leader location isn't updated by 1 . This local limitation count is used to keep track of how many cycles the local leader hasn't been changed.

DPLL: The local limitation counter is used in this phase to see if there is any chance of convergence rate or stasis within a group to reinitialize community politicians for potential solution space updates.

DPGL: It employs the worldwide limit count to determine if there is a risk of early converging or stability in the whole population, allowing the population to be partitioned into groups.

The proposed ISMO method begins with an initiation phase where the number of hosts or virtual machines is randomly assigned. The accessibility of hosts or virtual machines (VMs) for task performance is measured by fitness. If the number of tasks to be handled increases the hosts of VMs available at right state. In addition, determine the total of hosts or virtual machines into groups depending on their level of accessibility, which fluctuates in response to a rate of arriving work. The subgroups are re-initialized using the new value of fitness until the established good has met the number of iterations.

\section{RESULT AND DISCUSSION}

The present section evaluates the results of the proposed ISMO method for VM consolidation in the cloud's data centers is discussed. The proposed ISMO method's validation is carried out by using the larger data files that need to be stored in the servers of the cloud. In this research, the proposed work is compared with the benchmark models for VM in cloud data centers. In the proposed ISMO method is simulated using Java with system requirements like Windows 10 Operating System, RAM of 128GB, Intel Core i9 Processor, and Hard Disk of 4TB. The parameters considered to assess the ISMO method for VM consolidation for cloud data are explained in this section. The quantitative and comparative process of the proposed ISMO based on the existing method will be described in the section.

Published By:

Blue Eyes Intelligence Engineering and Sciences Publication

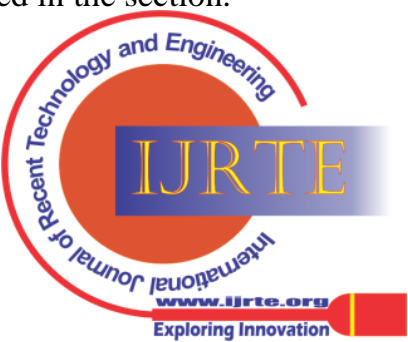




\section{A. Performance Metrics}

The proposed ISMO is used for evaluation and is compared with the existing method for performing VM consolidation in cloud data. The parameters are evaluated based on energy consumption, Performance Degradation due to Migration (PDM), SLA and Number of VM migration are employed for evaluating the effectiveness of the ISMO method with existing methods which are discussed as follows:

\section{- SLA Violation}

SLA is a concordance between a service provider and service user to particular services. It is determined using equation (4).

$$
S L A V=S V T A H * P D M
$$

(4)

\section{- Energy Consumption:}

Energy Consumption is the usage of data from the shared pool of computing resources. It is calculated by using equation (5).

$$
e(t)=\int_{t}^{0} P(t)
$$

\section{- VM migration:}

VM migration is the movement of virtual machines from a particular environment to other.

\section{- PDM:}

Equation (6) gives degradation in the performance because of migrations, in which the number of VMs data center as ' $\mathrm{m}$ '. The ' $C_{d y}$ ' predicted the PDM VMs and the total CPU utilization is requested based on the VM.

$$
P D M=1 / M \sum_{y=1}^{m} \frac{C_{d y}}{C_{r y}}
$$

\section{- Energy Efficiency:}

The energy efficiency of the system is estimated utilizing equation (7).

Energy Efficiency =

$$
\frac{1}{\text { Energy Consumption } * \text { SLA * Memory }}
$$

\section{B. Quantitative Analysis}

The results obtained in the proposed ISMO method about energy consumption, the efficiency of energy, Number of VM migration, SLA, and PDM based on the cloud's data VM consolidation are tabulated in data centers is as shown in the table 1 .

Table- I: The quantitative analysis for the proposed ISMO method evaluated in terms of Energy Efficiency, Energy consumption and SLA.

\begin{tabular}{|l|l|l|l|}
\hline Algorithms & $\begin{array}{l}\text { Energy } \\
\text { efficiency }\end{array}$ & $\begin{array}{l}\text { Energy } \\
\text { consumption } \\
\text { (KWh) }\end{array}$ & $\begin{array}{l}\text { SLA } \\
\text { violations } \\
(10-7)\end{array}$ \\
\hline NPA & - & 2410.8 & - \\
\hline DVFS & - & 803.91 & - \\
\hline THR-MMT-1.0 & 38 & 99.95 & 2613 \\
\hline THR-MMT-0.8 & 170 & 119.4 & 492 \\
\hline
\end{tabular}

\begin{tabular}{|l|l|l|l|}
\hline MAD-MMT-2.5 & 169 & 114.27 & 518 \\
\hline IQR-MMT-1.5 & 166 & 117.08 & 514 \\
\hline KAM-MMS-2.0 & 9231 & 83.33 & 13 \\
\hline KAI-MMS-1.0 & 6393 & 104.28 & 15 \\
\hline KMI-MRCU-1.0 & 28484 & 87.77 & 4 \\
\hline KMIMR-MRCPMB-1.0 & 30089 & 78.98 & 1 \\
\hline Proposed ISMO Method & 28266 & 28.5 & 1 \\
\hline
\end{tabular}

Table 1 shows the proposed ISMO method based the VM for cloud data centers with regard to SLA, energy efficiency and consumption of energy. Quantitative analysis is carried out using an existing algorithm to verify the effectiveness of the proposed ISMO algorithm. The energy efficiency of the proposed ISMO method is achieved 28266 whereas, the existing KAI-MMS-1.0 algorithm showed an energy efficiency of 6393. Similarly, the proposed ISMO method achieved energy consumption of 28.5 KWh and the existing KAI-MMS-1.0 showed energy consumption of 104.28 KWh. Then, the SLA violation of the proposed ISMO method is 1 and the existing KAI-MMS-1.0 showed an SLA violation of 15. So, the quantitative analysis showed that the proposed ISMO method achieved effective performance than the existing method. The pictorial representation of the proposed ISMO method for VM consolidation in cloud's data centers, with regard to the efficiency of energy, SLA, and consumption of energy is shown in figure 2.

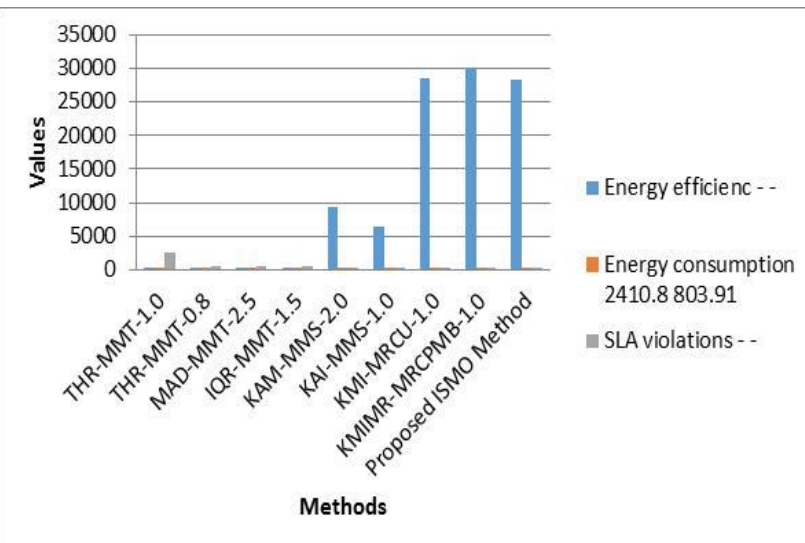

Figure II: The quantitative analysis of proposed ISMO method with respect to energy efficiency, energy consumption and SLA.

\begin{tabular}{|c|c|c|c|}
\hline Algorithms & SVTAH (\%) & PDM (\%) & $\begin{array}{l}\text { Number o } \\
\text { VM } \\
\text { migrations }\end{array}$ \\
\hline NPA & - & - & - \\
\hline DVFS & - & - & - \\
\hline THR-MMT-1.0 & 26.97 & 0.1 & 19852 \\
\hline THR-MMT-0.8 & 4.99 & 0.1 & 26567 \\
\hline MAD-MMT-2.5 & 5.24 & 0.1 & 25923 \\
\hline IQR-MMT-1.5 & 5.08 & 0.1 & 26420 \\
\hline KAM-MMS-2.0 & 1.73 & 0.01 & 6808 \\
\hline KAI-MMS-1.0 & 2.03 & 0.01 & 7519 \\
\hline KMI-MRCU-1.0 & 0.90 & 0.004 & 2821 \\
\hline KMIMR-MRCPMB-1.0 & 0.76 & 0.025 & 2608 \\
\hline Proposed ISMO Method & 0.7 & 0.015 & 2528 \\
\hline
\end{tabular}

Table- II: Quantitative analysis of proposed ISMO method in terms of SVTAH, PDM and Number of Migration 
Table 2 shows qualitative analysis of the proposed ISMO method based the VM for cloud data centers with regard to a number of VM migrations, SVTAH along PDM. The quantitative analysis is carried out using the existing algorithm so that the effectiveness of the proposed ISMO algorithm gets verified. The SVTAH of the proposed ISMO method is achieved 0.7 whereas, the existing KAI-MMS-1.0 algorithm showed an SVTAH of 2.03. Similarly, the proposed ISMO method achieved a PDM of 0.015 and the existing KAI-MMS-1.0 showed a PDM of 0.01.

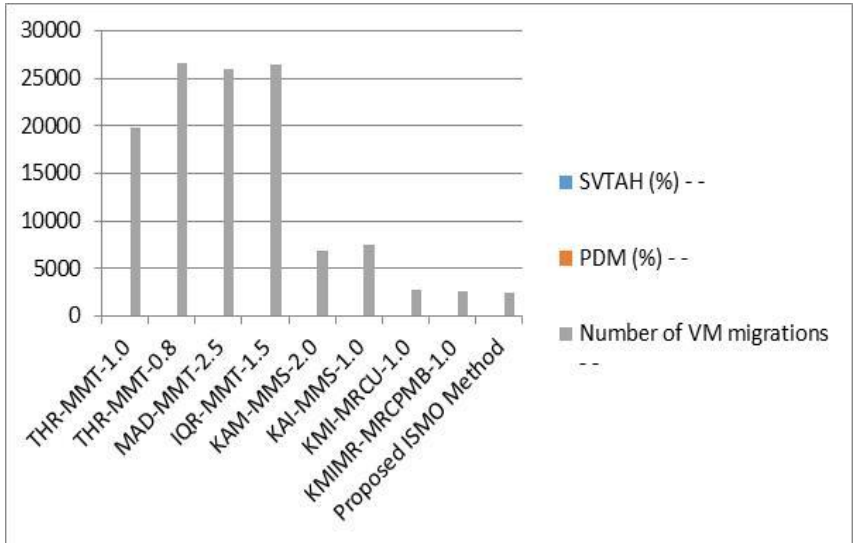

\section{Figure III: The quantitative analysis graph of the proposed ISMO method about a number of VM migrations, SVTAH and PDM.}

Then, the number of VM migration of the proposed ISMO method is 2528 and the existing KAI-MMS-1.0 showed VM migration of 7519 . So, the quantitative analysis showed that the proposed ISMO method achieved effective performance compared with the existing methods. The pictorial representation of the proposed ISMO method for VM consolidation in cloud's data centers, about number of VM migrations, SVTAH along with PDM, is shown in figure 3.

\section{Comparative Analysis}

The proposed ISMO results are compared with the previous existing models such as Joint VM Container Multi-criteria Migration Decision (JVCMMD) [12] and Adaptive Threshold VM Consolidation Framework [14] for the dynamic and secure public auditing of user data in the cloud with the different sizes from 100 to 100 in bits in terms of encryption and decryption time. The encryption time comparison with existing methods is given in table 3 .

Table 3 shows the comparison of the ISMO for cloud data center VM consolidation regard the energy efficiency, SLA violations, consumption of energy and VM migrations. The energy efficiency of the proposed ISMO method is achieved 28266 whereas, the existing algorithm showed an energy efficiency of 6009 and 10001. Similarly, the proposed ISMO method achieved an energy consumption of 28.5 and the existing method showed energy consumption of 48.92 and 59.89. Similarly, the SLA Violations of the proposed ISMO method achieved 1. Whereas, the existing method showed SLA violations of 28 and 23. Then, the number of VM migration of the proposed ISMO method is 2528 and the existing method showed VM migration of 5789 and 110999. So, the comparative analysis showed that the proposed ISMO method achieved effective performance in contrast to the existing method. The pictorial representation of the proposed ISMO method for VM consolidation at the cloud data centers improves the efficiency in terms of SLA violations, efficiency of energy, consumption of energy, and VM number is shown in figure 4.

Table- III: The comparison table of proposed ISMO method with the existing method.

\begin{tabular}{|l|l|l|l|l|l|}
\hline Authors & Methods & $\begin{array}{l}\text { Energy } \\
\text { efficiency }\end{array}$ & $\begin{array}{l}\text { Energy } \\
\text { consumption } \\
\text { (KWh) }\end{array}$ & $\begin{array}{l}\text { SLA } \\
\text { violations } \\
\text { VM migrations }\end{array}$ \\
\hline Gholipour et.al.[12] & $\begin{array}{l}\text { Joint VM Container Multi-criteria } \\
\text { Migration Decision (JVCMMD) }\end{array}$ & 10001 & 48.92 & 28 & 5789 \\
\hline Khattar et.al.[16] & $\begin{array}{l}\text { Adaptive Threshold VM } \\
\text { Consolidation Framework }\end{array}$ & 6009 & 59.89 & 23 & 110999 \\
\hline Proposed & ISMO method & 28266 & 28.5 & 1 & 2528 \\
\hline
\end{tabular}

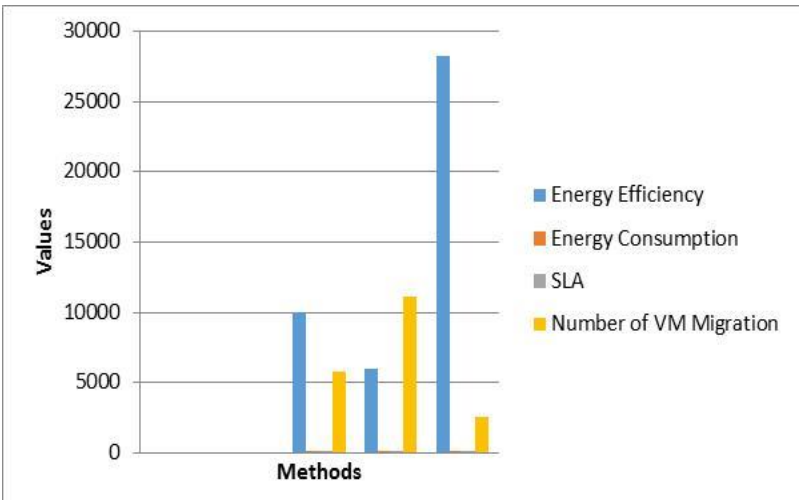

Figure IV: The comparison graph of the proposed ISMO method for VM consolidation in the cloud's data centers with the existing method

\section{CONCLUSION}

Growing usage of cloud computing shows the development with a massive data centers required energy. An energy efficiency is improved at the data centers can help cloud providers enhance their Return on Investment (ROI) while lowering greenhouse gas emissions. Despite the increase in the prevalence of containers data centers, the energy efficiency of resources management techniques' is still a concern. To solve such an issue, the ISMO algorithm for cloud center optimization is proposed.

Published By:

Blue Eyes Intelligence Engineering and Sciences Publication

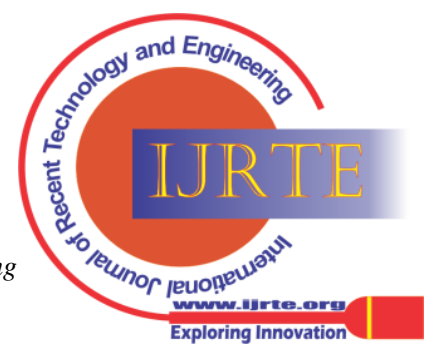


The VM consolidation framework based on the proposed ISMO algorithm decreases consumption of energy and tries to curtail Service Level Agreement violations, simultaneously. The approach is utilized to provide energy-efficient computing in cloud data centers. The comparative and quantitative analysis showed that the proposed approach performed well. In the future, the heuristic techniques can be used for both VM and container reduction challenges.

\section{REFERENCES}

1. Y. Saadi, and S. El Kafhali, "Energy-efficient strategy for virtual machine consolidation in cloud environment," Soft Computing, vol. 24, 2020, pp. 14845-14859.

2. S. Y. Z. Fard, M. R. Ahmadi, and S. Adabi, "A dynamic VM consolidation technique for QoS and energy consumption in cloud environment," The Journal of Supercomputing, vol. 73, 2017, pp 4347-4368.

3. M. Tarahomi, M. Izadi, and M. Ghobaei-Arani, "An efficient power-aware VM allocation mechanism in cloud data centers: a micro genetic-based approach," Cluster Computing, vol. 24, 2020, pp. 919-934.

4. A. Tarafdar, M. Debnath, S. Khatua, and R. K. Das, "Energy and quality of service-aware virtual machine consolidation in a cloud data center," The Journal of Supercomputing, vol. 76, 2020, pp. 9095-9126.

5. P. Geetha, and C. R. Robin, "Power conserving resource allocation scheme with improved QoS to promote green cloud computing," Journal of Ambient Intelligence and Humanized Computing, vol. 12, 2020, pp. 7153-7164.

6. M. A. Gomez-Rodriguez, V. J. Sosa-Sosa, J. Carretero, and J. L. Gonzalez, "CloudBench: an integrated evaluation of VM placement algorithms in clouds," The Journal of Supercomputing, vol. 76, 2020, pp. 7047-7080.

7. J. Son, A. V. Dastjerdi, R. N. Calheiros, and R. Buyya, "SLA-aware and energy-efficient dynamic overbooking in SDN-based cloud data centers," IEEE Transactions on Sustainable Computing, vol. 2, 2017, pp. 76-89.

8. A. Kaur, A. Diwakar, and R. Vashisht, "Alternatives to VM consolidation techniques for energy aware cloud computing," 2017 International Conference on Advances in Computing, Communications and Informatics, 2017, pp. 2005-2009.

9. M. Abdullah, K. Lu, P. Wieder, and R. Yahyapour, "A heuristic-based approach for dynamic vms consolidation in cloud data centers," Arabian Journal for Science and Engineering, vol. 42, 2017, pp. 3535-3549.

10. M. Ranjbari, and J. A. Torkestani, "A learning automata-based algorithm for energy and SLA efficient consolidation of virtual machines in cloud data centers," Journal of Parallel and Distributed Computing, vol. 113, 2018, pp. 55-62.

11. J. Liu, S. Wang, A. Zhou, J. Xu, and F. Yang, "SLA-driven container consolidation with usage prediction for green cloud computing," Frontiers of Computer Science, vol. 14, 2020, pp. 42-52.

12. N. Gholipour, E. Arianyan, and R. Buyya, "A novel energy-aware resource management technique using joint $\mathrm{VM}$ and container consolidation approach for green computing in cloud data centers," Simulation Modelling Practice and Theory, vol. 104, 2020, pp. 102127.

13. A. Ibrahim, M. Noshy, H. A. Ali, and M. Badawy, "PAPSO: A Power-Aware VM Placement Technique Based on Particle Swarm Optimization," IEEE Access, vol. 8, 2020, pp. 81747-81764.

14. L. Li, J. Dng, D. Zuo, and J. Wu, "SLA-aware and energy-efficient VM consolidation in cloud data centers using robust linear regression prediction model," IEEE Access, vol. 7, 2019, pp. 9490-9500.

15. M. Gamal, R. Rizk, H. Mahdi, and B. E. Elnaghi, "Osmotic Bio-Inspired Load Balancing Algorithm in Cloud Computing," IEEE Access, vol. 7, 2019, pp. 42735-42744.

16. N. Khattar, J. Singh, and J. Sidhu, "An energy efficient and adaptive threshold VM consolidation framework for cloud environment," Wireless Personal Communications, vol. 113, 2020, pp. 349-367.

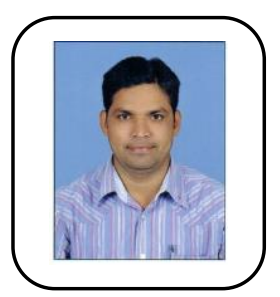

\section{AUTHORS PROFILE}

K. Prem Kumar is Ph.D. research scholar in Computer Science and Engineering at Jawaharla Nehru Technological University, Hyderabad, India. He obtained Master of Technology in Computer Science and Engineering from JNT University, Hyderabad in 2010 and B.Tech degree in Computer Science and Engineering from JNT University, Hyderabad in 2004. He is currently working as Assistant Professor in the Department of CSE at ACE Engineering College, Ghatkeshar, Hyderabad, India. He is having more than 12 years of experience in teaching and research. He published 4 research articles in reputed international conferences and journal. His current areas of interest include cloud computing, Distributed Systems and Software Engineering. He is a life member of ISTE.

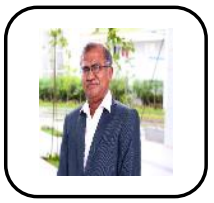

T. Ragunathan received his Ph.D in Computer Science and Engineering from IIIT, Hyderabad. He has got 26 years of teaching experience including 15 years of research experience. He is currently working as Professor and Head in Computer Science and Engineering Department at SRM University, AP, Amaravati, Andhra Pradesh. Dr. Ragunathan carries out research activities with regard to the development of fast distributed file system for efficient storage and retrieval of large data. He also carries out research works on task scheduling and load balancing issues in cloud computing systems.

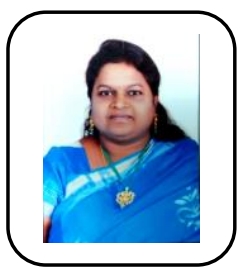

D.Vasumathi received her Ph.D in Computer Science and Engineering from Jawaharlal Nehru Technological University (JNTU), Hyderabad-India in 2011. She is currently working as Professor and Head of Department of Computer Science and Engineering at JNTUH college of Engineering Hyderabad (autonomous)-India. She is having more than 18 years of experience in teaching and research. She worked as various positions at Administrative levels such as Additional Controller of Examinations, General Secretary for Teaching Association, and President for Women Association at JNTU-Hyderabad. She has been working as a reviewer for reputed Journals, Conferences at National and International levels. She published more than 50 research articles at reputed Journals and Conferences. Under her leadership organized several FDPs, workshops and conferences at JNTUH. Her areas of interest include Data warehousing \& Data mining, Information Retrieval Systems, Bigdata Analytics, Cloud Computing and Network Security. She is Life member of ISTE and CSI.

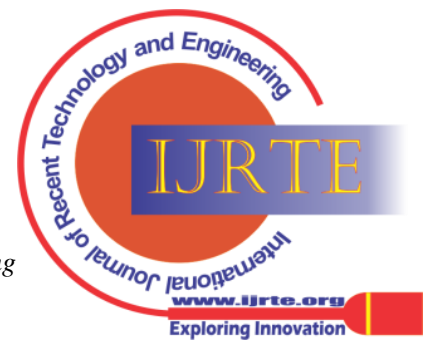

